\section{Scopolamine effects on locomotor and exploratory activity in rats ${ }^{1}$}

GARY WALTERS and R. G. BLOCK, University of Toronto, Toronto 5, Canada

Scopolamine did not affect the course of habituation in rats but resulted in an increase in both general locomotor and specific exploratory activity-the mognitude of this effect decreasing over test days. Locomotor and exploratory behavior were not differentiated by the drug.

Experiments with anticholinergic drug, such as scopolamine and atropine, have indicated that reduction in brain acetylcholine activity produces increased behavioral activity and attenuation of habituation to novel stimuli due to disinhibition (e.g., Carlton, 1963, 1966, 1968). The considerations which led to the present experiment were as follows: (1) Previous experiments in this area have not included measures of responding both under drug and in a subsequent test without drug, although both types of measures have been made in separate experiments. (2) Locomotor activity and specific exploration have been differentiated behaviorally (Berlyne, 1960) and physiologically by lesions (Glickman, Sroges, \& Hunt, 1960). Does scopalamine differentially affect these two types of behavior? (3) Glickman, Sroges, \& Hunt (1960) also suggested that activity in familiar and novel environments may be controlled by different brain mechanisms. Is scopolamine different in its effects in these two types of situations?

\section{SUBJECTS}

The Ss were 36 experimentally naive male hooded rats, weighing between 250 and $300 \mathrm{~g}$ and maintained in individual cages on ad lib food and water throughout the experiment.

\section{APPARATUS}

An apparatus was designed to permit the separation of locomotor activity from specific exploratory responses. It consisted of a plywood box ( $18 \times 18 \times 18$ in.) with a sliding Plexiglas top. The floor was sectioned into four 9-in. squares, each mounted on springs so that depression of a square closed a microswitch. The floor was covered with a sheet of dark green polyethylene. In each corner, at floor level, was a $2 \frac{1}{2} \times 2^{1 / 1} / 2 \times 3$ in. long cubicle containing novel objects-a two-proriged wall plug, a double banana plug, a microswitch, and a spring. These cubicles could be set open or closed. They were just large enough so that no other part of the rat's body except the head could be inserted. A head insertion operated a photo-cell relay. Number of entries into each cubicle (as the measure of specific exploratory activity) and number of entries into each floor square (as the measure of locomotor activity) were recorded on an Esterline-Angus event recorder. The inside of the box was painted flat black, and the inside of the cubicles, a semi-gloss white. The cubicles were lighted by the 6.3-VAC photo-cell light source, and the whole apparatus was lighted from above by a 15-W bulb. Masking noise was provided by a white-noise generator.

\section{PROCEDURE}

IP injections were given $20 \mathrm{~min}$ before an animal was tested. All rats received $1 \mathrm{ml}$ of isotonic saline per kilogram of body weight on all days. Drug-group animals received $1 \mathrm{mg} / \mathrm{kg}$ of scopolamine hydrobromide dissolved in saline. Animals we re assigned to one of four groups; the following designations indicate whether the animals received saline or drug and whether the cubicles containing the novel objects were open or closed: ND-NS (no drug, no stimuli), ND-S (no drug, stimuli), D-NS (drug, no stimuli), D-S (drug, stimuli).

Animals were run individually once daily for $15 \mathrm{~min}$. In Stage 1 (familiarization), all (drug/stimuli) the animals were treated according to their group designation, except that no drug was given every second day. This drug/no-drug alternation pattern was repeated three times for a total of 6 days. Stage 3 (drug/all groups stimuli) was a continuation of Stage 2, but with all groups exposed to the novel stimuli. This stage consisted of one drug day and one no-drug day. Stage 3 was not originally planned, but was added to test hypotheses to account for unexpected results.

\section{RESULTS}

All groups showed a decline in locomotor activity both within and across days in Stage 1. There were no significant differences among groups during this stage. Figure 1 shows mean locomotor activity in Stage 2. Exploratory activity, shown in Fig. 2, was very similar to locomotor activity, so that there was no evidence for these two types of behavior being differentially affected. It can be seen from Figs. 1 and 2 that the drug groups were consistently higher in total response output, but that the drug did not retard habituation either within or across days. It is also evident from Figs. 1 and 2 that there was no difference among groups on no-drug days. These observations were confirmed statistically by analysis of variance.

Stage 3 was added to test the possibility that the decline over days of responding in the drug groups was due to development of drug tolerance, side-effects, or fatigue. If such was the case, the group which received animals were given saline and were run with the cubicles closed for 5 days. In Stage 2

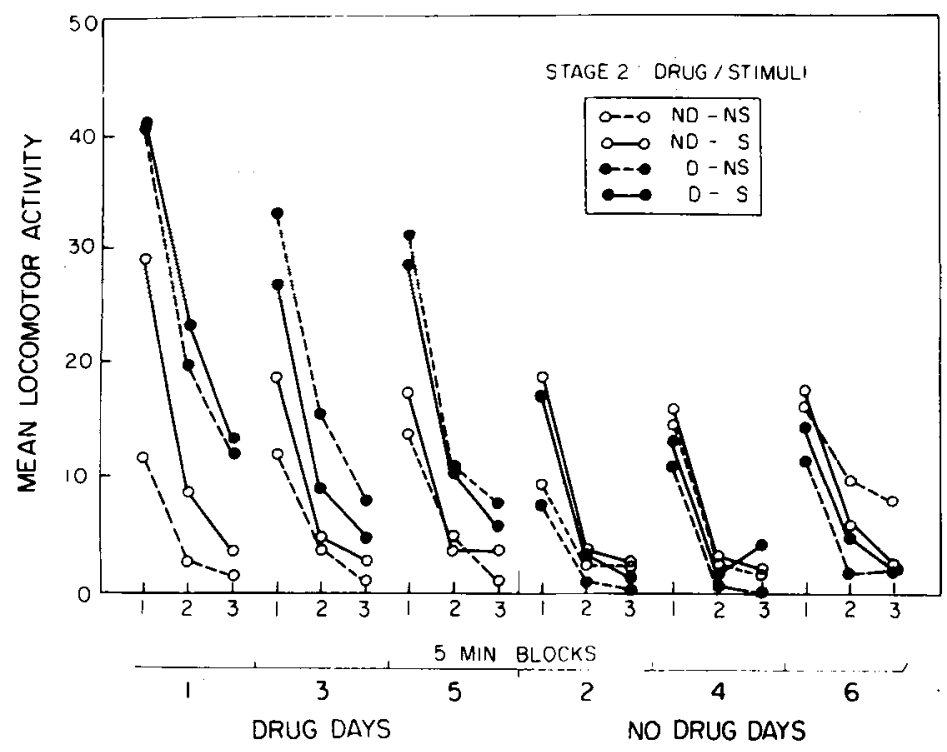

Fig. 1. Mean locomotor activity during Stage 2, when treatments were introduced. Drug-days performance is shown at the left; no-drugdays performance is shown at the right. 


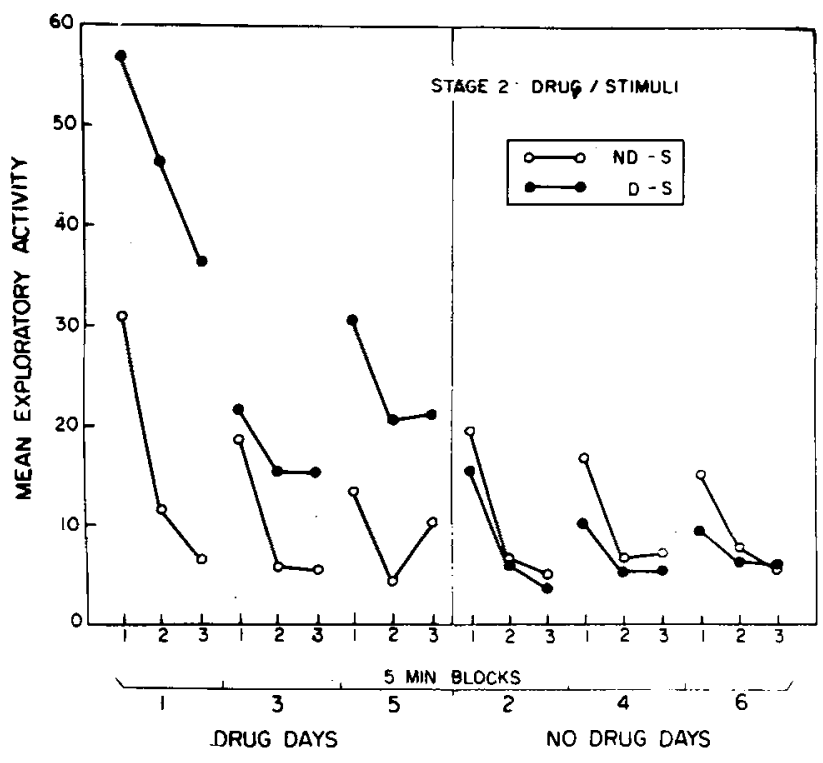

Fig. 2. Mean exploratory activity during Stage 2. Drug-days performance is shown at the left; no-drug-days performance is shown at the right.

drug but was not previously exposed to the stimuli should have suffered from similar drug effects as the drug group which was exposed to the stimuli and its level of responding in this stage should have been similar to the previously exposed group. The results are shown in Fig. 3. Both groups, drug and no-drug, newly exposed to the novel stimuli showed increased responding over their scores on the previous day and over the other two groups. Effects of newly introducing the stimuli were significant beyond the .01 level for both locomotor and exploratory activity $(F=10.85$ and 9.51 , respectively, both with $1 / 32 \mathrm{df}$ ). There were no significant differences among groups on Day 2, when drug was not given.

\section{DISCUSSION}

The effect of scopolamine in this experiment was to increase both locomotor and specific exploratory activity. There was no evidence for attenuation of habituation nor of an effect of drug-day performance on interpolated no-drug days. The Stage 3 data indicate that decline in responding under drug was due to habituation rather than to deleterious drug effects.

Our results must be qualified by the following considerations: (1) It is impossible to tell whether habituation of animals across drug days was due to performance on drug days, performance on no-drug days, or both. When the experiment was designed, such habituation was not expected and therefore not controlled. (2) The use of several drug dosages was omitted from this experiment, but should be included in any further work. Carlton (1965) found that scopolamine affected habituation, as measured by the degree of suppression of behavior by a stimulus, using $0.5 \mathrm{mg} / \mathrm{kg}$, while Douglas \& Isaacson (1966), using a spontaneous alternation measure, found an effect at $1.2 \mathrm{mg} / \mathrm{kg}$, but not at lower doses. (3) The validity of the head-poking response as a measure of specific exploration may be questioned. It may have been that scopolamine activated this particular response without its being related to the specific stimuli employed. Future studies should make the presentation of the novel stimuli contingent upon the response, as, for example, in photic reinforcement experiments.

Keeping these considerations in mind, the results can still be generally viewed as not offering support for previous findings of attenuation of habituation by scopolamine, but consistent with findings of increased activation.

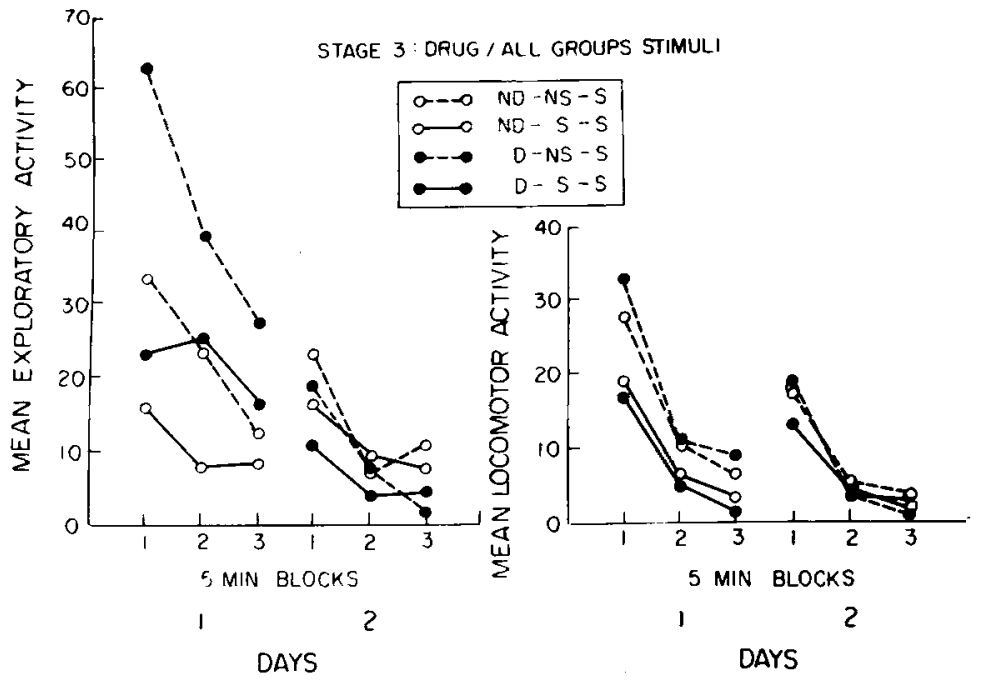

Fig. 3. Mean exploratory activity (left) and mean locomotor activity (right) during Stage 3 . All groups were exposed to the stimuli during this stage. Drug was given to the two drug groups on Day 1 , and saline was given to all groups on Day 2.

\section{REFERENCES}

BERLYNE, D. E. Conflict, arousal and curiosity. New York: McGraw-Hill, 1960.

CARLTON, P. L. Cholinergic mechanisms in the control of behavior by the brain. Psychological Review, 1963, 70, 19-39.

CARLTON, P. L. Scopolamine, amphetamine and light-reinforced responding. Psychonomic Science, 1966, 5, 347-348.

CARLTON, P. L. Brain-acetylcholine and habituation. In P. B. Bradley and M. Fink (Eds.), Progress in brain research. Vol. 28. New York: Elsevier, 1968. Pp. 48-60.

DOUGLAS, R. J., \& ISAACSON, R. L. Spontaneous alternation and scopolamine. Psychonomic Science, 1966, 4, 283-284.

GLICKMAN, S. E., SROGES, R. W., \& HUNT, J. Brain lesions and locomotor exploration in the albino rat. Journal of Comparative \& Physiological Psychology, 1964, 58, 93-100. NOTE

1. This work constituted the BA research thesis of R.G.B. and was assisted by grants to G.C.W. from the National Research Council of Canada (APA-161) and the Ontario Mental Health Foundation (OMHF No. 107). 